\title{
Titan as an analog of Earth's past and future
}

\author{
J.I. Lunine ${ }^{a}$ \\ Lunar and Planetary Laboratory, The University of Arizona, Tucson, AZ 85721, USA
}

\begin{abstract}
Titan, the second-largest moon in the solar system, is revealed by the joint ESA-NASA mission Cassini-Huygens to have a dense atmosphere of nitrogen and methane, lakes and seas of liquid methane and ethane, evidence for fluvial (river-based) erosion, and vast equatorial expanses of dunes made of organic material. It also has an ocean, probably mostly of water, beneath its icy crust. The presence of organic material and episodes of melting of the icy crust or breaching to reveal the ocean beneath provides the possibility of chemistry replicating that leading to the origin of life, on scales of time and space much larger than can be achieved in the laboratory. In this sense Titan may replicate aspects of Earth's past. But the methane cycle, which operates in the absence of a massive ocean covering Titan's surface, more resembles the kind of hydrological cycle Earth might have in the far future as the brightness of the Sun increases and water begins to escape rapidly.
\end{abstract}

\section{Introduction}

Titan is Saturn's largest moon, and is only slightly smaller than the largest moon of the solar system, Ganymede ( $5,260 \mathrm{~km}$ vs. $5,150 \mathrm{~km})$. Both bodies have a bulk density of $1.9 \mathrm{~g} / \mathrm{cm}^{3}$. Examination of the list of abundances of the elements [1] indicates that water ice, silicates and iron dominate as solids, so putting together ice with a silicate-metal mix typical of primitive meteorites [2] yields bodies that are 55-65\% rock with most of the rest water ice (and for Titan, sulfur in some models; [3]). Thus, Ganymede and Titan are rock-ice worlds. But what distinguishes these two Mercury-sized moons from each other is that Titan has an atmosphere - a dense one. The atmosphere, $95 \%$ nitrogen and $5 \%$ methane near the surface, has a pressure 1.5 times that of Earth's at a temperature (dictated by the great distance from the Sun) of $94 \mathrm{~K}$. This makes the air density at the surface approximately four times that at sea level on Earth.

Methane in Titan's atmosphere is key to understanding how the surface and atmosphere work, analogously to - but with important differences from - the role of water on the Earth [4]. In this paper the role of methane on Titan as it is understood today will be described. The implications for a comparative study of Titan and the Earth will then be considered from two points of view: Earth's past and future. Chemically Titan's surface and atmosphere provide perhaps the best stage in the solar system for reactions that might replicate the chemistry that led to the origin of life on Earth billions of years ago. From the point of view of climate, the methane cycle perhaps gives us a hint of what Earth's water cycle will look like in the far future as increasing luminosity of the Sun changes the stability of water in the oceans and the atmosphere. These two points of comparison between Titan and our home planet's past and future will be considered in the next two sections of the paper. The final section briefly describes future exploration plans.

Most of what we know of Titan comes from the successful Cassini-Huygens mission, a joint European and United States mission which began operations at Saturn in 2004 [5]. The

\footnotetext{
a e-mail: jlunine@lpl.arizona.edu
}

Article published by EDP Sciences and available at http://www.epj-conferences.org or http://dx.doi.org/10.1140/epjconf/e2009-00926-7 
literature on Titan is thus growing rapidly, and no attempt is made here to provide a complete list of citations. The reader should consult recent compendia [6] and indices of the major journals such as Science and Nature, to find the latest discoveries and interpretations.

\section{Titan's methane cycle}

A number of processes have been identified in Titan's atmosphere and on its surface by Voyager, ground-based and Cassini-Huygens data (Fig. 1). Solar ultraviolet radiation and energetic particles from the Saturnian magnetosphere rain down on the atmosphere [7], converting methane to higher hydrocarbons and nitriles, primarily [8]. These form aerosols and rain out on the surface, with ethane and propane forming liquids while the other products are stable solids (that should slightly dissolve in the liquid phase). If the chemistry has gone on over the age of the solar system the solid and liquid deposits could be thick - hundreds of meters globally - though Cassini and Huygens data suggest smaller volumes, at least on the surface [9]. In the lower atmosphere, a fraction of the sunlight - principally in the red part of the spectrum - falls on the surface, and absorption of the corresponding outward infrared radiation by opacity generated through collisions among nitrogen, methane and hydrogen creates a significant greenhouse warming of about 10-15 degrees at the surface. The temperature profile is thus not very different in shape from that of the Earth [10], though with a shallower tropopause and a larger physical extent thanks to Titan's lower gravity. Dunes made of organic solids cover much of Titan's equatorial region [11], except for a continent-sized area called Xanadu which is characterized by hills and small mountains, incise by broad river valleys. At the high latitudes dark areas that are likely lakes of ethane and methane exist [12], while at intermediate latitudes a number of impact craters and possible candidate features associated with "cryovolcanism" (see below) are found. Fluvial erosion at the landing site of the ESA Huygens probe [13] suggests that heavy methane rains occur every so often. The ice-rock interior of Titan may hold substantial amounts of methane or carbon dioxide; the extent to which it is differentiated into a rock core and ice mantle is yet to be determined. Hints exist [14] of a liquid water layer below the crust.

Cassini-Huygens data have considerably clarified the methane cycle on Titan (Fig. 2). Evidently the equatorial region is at present too dry for lakes, unlike the poles, but the presence of stream channels in the hills behind the Huygens landing site suggests torrential methane rains occur at certain times. Because of the weak amount of sunlight, the relative humidity at the landing site of about $45 \%$ is insufficient to initiate rainstorms [15], and simple energy balance considerations suggest that centuries or millennia may be required to remoisten the equatorial atmosphere with sufficient amounts of methane. However, the source of such methane has yet to be identified. The northern hemisphere lakes are insufficient, for reasonable estimates of their volume, by a factor of two, but if they are connected to an underground methane table (or methane-ethane table), akin to aquifers on Earth, then the moist polar regions might indeed be the source for equatorial and mid-latitude fluvial erosion.

\section{Titan and Earth's past}

The organic chemistry ongoing in Titan's atmosphere produces mostly higher hydrocarbons (ethane, acetylene, and others with carbon to hydrogen ratios larger than methane) and nitriles. Oxygen precipitating into Titan's atmosphere, derived ultimately from water ice emitted by the smaller Saturnian moon Enceladus [17], shunts a small amount of carbon into carbon monoxide and carbon dioxide [18].

By itself, the stratospheric photochemistry is not particularly interesting from the point of view of prebiotic chemistry because of the almost total lack of oxygen, and because the presence of a temperature minimum (tropopause) forces species to condense in the form of aerosols before they can become very complex.

But high above the stratosphere, energetic particles from the Saturnian magnetosphere energize a chemical reaction network that produces complex polymers detected by the Cassini mass 


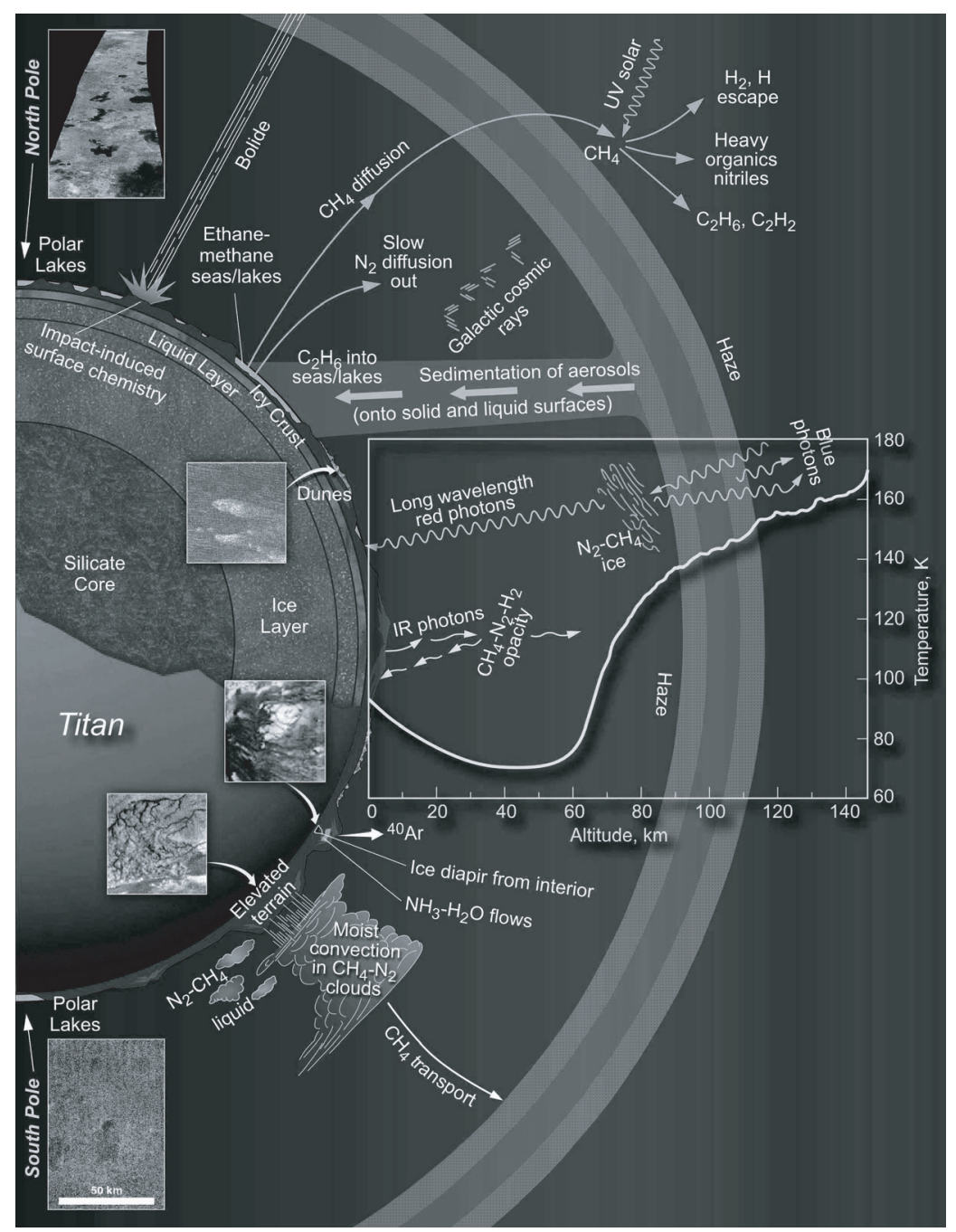

Fig. 1. Schematic illustration of the connections among Titan's interior, surface, atmosphere, and cosmic environment. The temperature profile is based on Voyager data, but is representative of what Cassini found as well. Images show lakes at north and south poles, midlatitude terrains with dunes, and fluvial features carved in the ice crust. Caltech/JPL artwork based on an original figure in [16] with images from the Cassini and Huygens probes added.

spectrometer. And at the surface, the aerosols that sediment out of the atmosphere potentially come into contact with water ice that probably forms the bulk of the crust of the moon. While chemical reactions with water ice at $94 \mathrm{~K}$ are very slow, and there is limited contact between the ice and the organics anyway, the exciting possibility is that liquid water might be present in certain times and places allowing aqueous organic chemistry to occur.

Such aqueous chemistry requires sources of energy to melt the ice, because solar heating falls far short at 9.5 times the Earth-Sun distance. Large-scale impacts of cometary and asteroidal bodies have occurred throughout Titan's history, and these are capable of melting large volumes of the ice crust for timescales of centuries to millennia [19]. In some cases organic molecules are only lightly shocked in the impact, and fall into the water melt zone where aqueous chemistry could take place.

Another source of energy for melting water ice is the heat within Titan, generated during formation, as well as heat generated by the decay of radioactive elements within the rocky part of the moon. This, a kind of ice volcanism (more usually called "cryovolcanism"), almost 


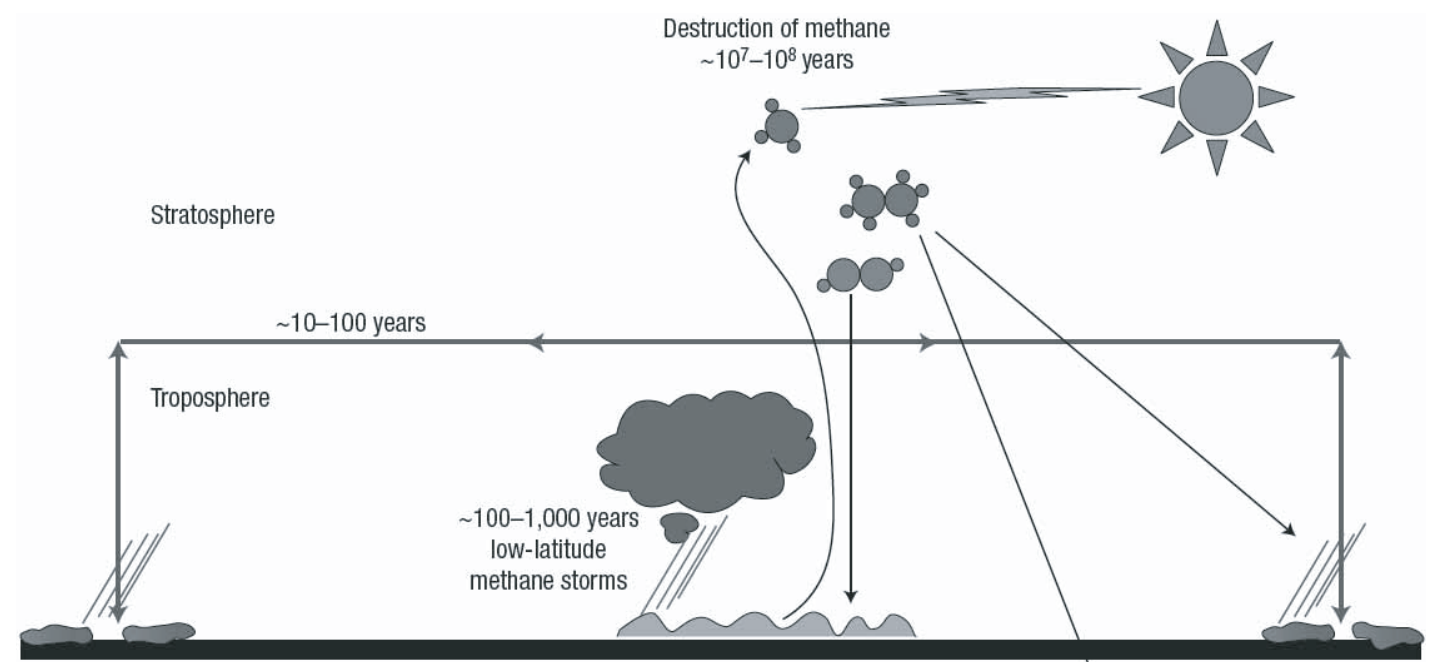

Fig. 2. The methane cycle on Titan, adapted from [4], is shown with rough timescales for the various processes. Methane is either primordial or made in Titan's interior and then is outgassed to the surface. It cycles from pole-to-pole, perhaps resident at high latitudes (left and right sides of figure) in lakes. Periodically the equatorial humidity increases to the point that convective storms at low latitudes (center of figure) are possible, carving fluvial features. Photochemistry and charged particle chemistry produces higher hydrocarbons and nitriles, some of which ends up in the hydrologic system as ethane, and some as solids that agglomerate to form equatorial dunes. Ethane might be lost to the crust in voids or as clathrate hydrate.

certainly requires a low-melting point additive such as ammonia to allow the possibility of liquid to form while the surrounding ice remains hard enough not to soften and convect the heat away, and to lower the density of liquid water to at least neutral buoyancy [20]. Titan's heat flow is about $8 \%$ that of the Earth's [2], which is plenty for extensive cryovolcanism, and Cassini radar images of Titan's surface hint at the possibility of "cryo" lava flows [21]. Again, for larger flows, lifetimes of survival of liquid water in the flows could be decades or more, with ammonia present, and such flows would have covered up and then incorporated deposits of organic molecules on the surface manufactured in the atmosphere.

Literally one cannot predict accurately the products of aqueous reaction of oxygen-poor organic molecules with water over large spatial and temporal time scales, because experiments are greatly limited by comparison, however carefully performed [22]. Instead, one must assert that under appropriate conditions, perhaps the aqueous reactions produce amino acids and nucleic acid bases, along with sugars [19]. But are the products random, that is, small amounts of biologically-relevant amino acids and sugars in a soup of other less relevant products? Does a preferential handedness or enantiopurity - arise thanks to the large spatial and temporal scales? [23]. The only way to answer these questions would be to identify deposits of organics on Titan's surface that are in areas of cryovolcanic flows or impact craters and analyze them. If the nature of the altered organics were dramatically different from that obtained in a small-volume, brief-duration experiment in a laboratory organic reactor, one might then ponder whether large spatial and temporal scales enabled chemistry akin to that which might have occurred in particular environments on the early Earth.

The question has been raised as to whether our view of Titan with respect to organic chemical evolution toward life might be too conservative, and whether indeed a form of life or at least highly organized organic chemistry might occur in liquid methane and ethane [24]. If so - and there is no detailed chemical model for such a life form, nor is there a convincing proof that such is impossible - its home would be in the ethane-methane lakes and seas of the high northern latitudes on Titan (Fig. 3). Discovery of a form of chemistry sufficiently organized that it might be called life would change the nature of the search, not only in our solar system but in other planetary systems where one would have to consider tow habitable 


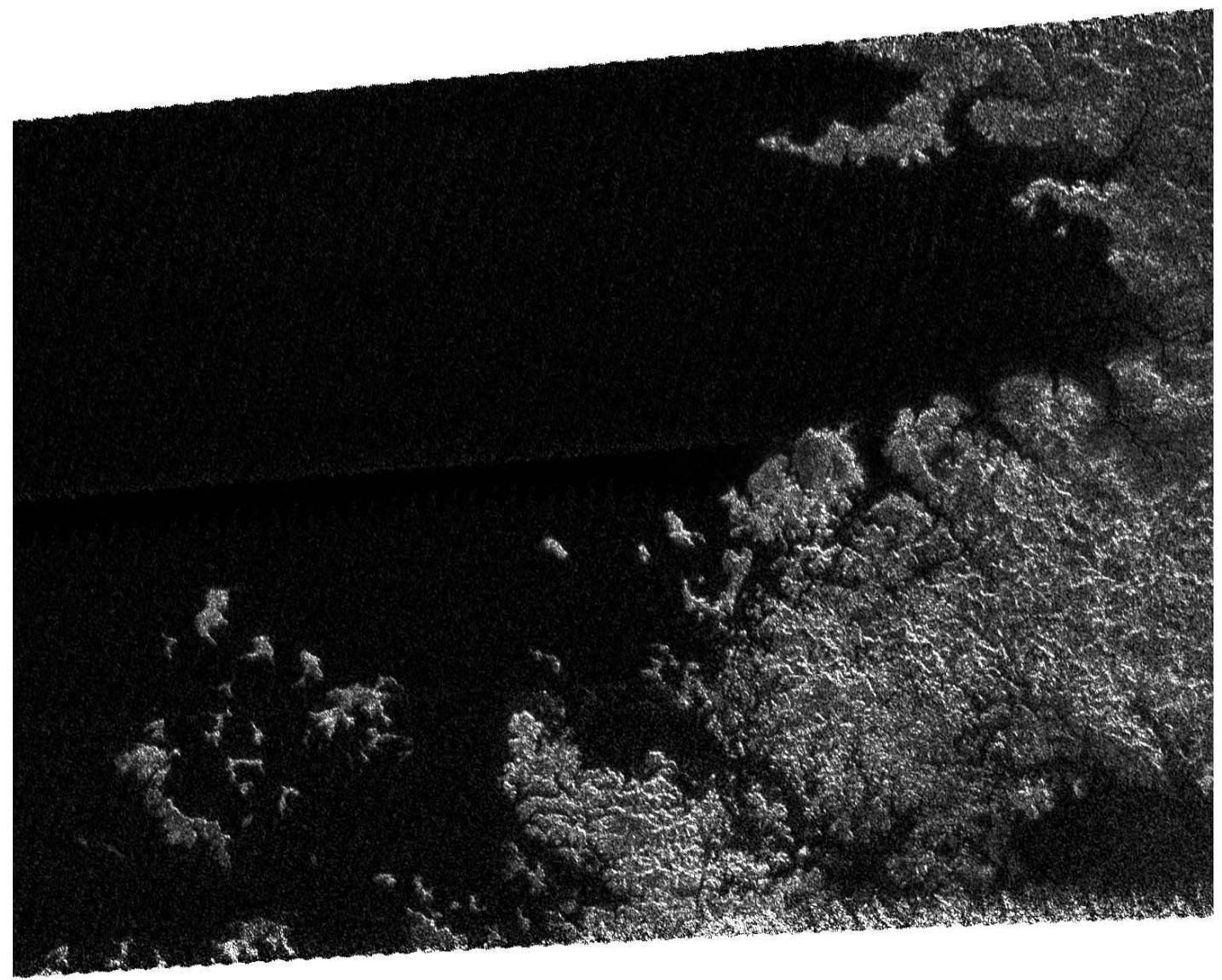

Fig. 3. Portion of a large area seen in radar as very dark, interfingering with hills and valleys apparently carved by liquid. Based on spectra in illuminated areas [25] and radar data, the dark area is likely liquid methane and ethane some tens of meters or more deep [26]. The image is about 160 kilometers by 270 kilometers at 300 -meter resolution. The image is centered near 70 degrees north latitude on Titan. Courtesy NASA-JPL.

zones - one for water and one for methane. Indeed, a committee of the National Academy of Sciences wrote "Thus the environment of Titan meets the absolute requirements for life. Titan is not at thermodynamic equilibrium. It has abundant carbon-containing molecules and heteroatoms and a fluid environment. Titan's temperature is low enough to permit a wide range of bonding, covalent and noncovalent. Titan undoubtedly offers other resources believed to be useful for catalysis necessary for life, including metals and surfaces." [27].

\section{Titan and Earth's future}

Titan is also an analog, albeit with different working materials, of a possible future state of the Earth when surface conditions may preclude stable equatorial/mid-latitude oceans. Over the history of the Earth our planet's hydrologic cycle has been stable, dipping a few times into deep ice ages when much of the ocean surface was frozen over, but never moving much in the other direction toward a state of large amounts of water vapor in the atmosphere. The temperature profile of the lower atmosphere - the troposphere - of the Earth drops steeply to a value between $190-210 \mathrm{~K}$ at the temperature minimum or tropopause - whose altitude varies seasonally and latitudinally from $8-17 \mathrm{~km}$ (consult any standard Earth atmosphere text 


\begin{tabular}{|c|c|c|}
\hline Decadal Survey p. 138 & Cassini & TSSM \\
\hline $\begin{array}{l}\text { What are the chemistry, } \\
\text { distribution and cycling of organic } \\
\text { materials on Titan? }\end{array}$ & $\begin{array}{l}\text { Methane/ethane sensed at } \\
\text { Huygens site; high altitude } \\
\text { polymers; lakes, fluvial }\end{array}$ & $\begin{array}{l}\text { High molecular weight mass } \\
\text { spectroscopy; hi-res imaging at } 5 \\
\text { microns; near-IR spectra }\end{array}$ \\
\hline $\begin{array}{l}\text { Is Titan internally active, producing } \\
\text { water-rich environments with } \\
\text { potential habitability? }\end{array}$ & $\begin{array}{l}\text { Spin rate evidence for ocean; radar } \\
\text { images of cryovolcanic features; } \\
\text { Near-IR spectra of carbon dioxide } \\
\text { patches }\end{array}$ & $\begin{array}{l}\text { Accelerometry-enhanced gravity; } \\
\text { hi-res surface imaging; surface } \\
\text { temp-erature monitoring in } \\
\text { thermal IR; 5-6 micron spectra }\end{array}$ \\
\hline $\begin{array}{l}\text { What are the current state and the } \\
\text { history of Titan's surface? }\end{array}$ & $\begin{array}{l}\text { Radar and VIMS show dearth of } \\
\text { craters; fluvial transport at Huygens } \\
\text { site }\end{array}$ & $\begin{array}{l}\text { High resolution imaging; radar } \\
\text { altimetry and sounding; near-IR } \\
\text { spectra }\end{array}$ \\
\hline $\begin{array}{l}\text { What drives the meteorology of } \\
\text { Titan? }\end{array}$ & $\begin{array}{l}\text { Huygens wind and CIRS } \\
\text { temperature data provide crude } \\
\text { basis for GCM }\end{array}$ & $\begin{array}{l}\text { Sub-millimeter wind/thermal } \\
\text { mapping; IR mapping; near-IR cloud } \\
\text { sounding }\end{array}$ \\
\hline $\begin{array}{l}\text { Has there been climate change on } \\
\text { Titan? }\end{array}$ & $\begin{array}{l}\text { Fluvial erosion at desert Huygens } \\
\text { site; extensive dunes; missing } \\
\text { ethane }\end{array}$ & $\begin{array}{l}\text { Hi resolution imaging; radar } \\
\text { sounding; near-IR spectra }\end{array}$ \\
\hline $\begin{array}{l}\text { Could Titan support life forms that } \\
\text { do not require liquid water? }\end{array}$ & $\begin{array}{l}\text { High latitude lakes found, as well as } \\
\text { environments where active fluvial } \\
\text { flow may occur, and cryo-volcanism }\end{array}$ & $\begin{array}{l}\text { Hi-res spectra over } 5-6 \text { microns; } \\
\text { repeat surface coverage; high } \\
\text { molecular weight sampling of } \\
\text { upper atmosphere organics }\end{array}$ \\
\hline
\end{tabular}

Fig. 4. Table showing how Cassini-Huygens (middle column) made discoveries that addressed questions raised in 2003 by the Decadal Survey [35], and how a future mission (right column) called TSSM (described in the text) will make observations to extend our knowledge of Titan further. Abbreviations and acronyms: CIRS is Cassini infrared radiometer and spectrometer; GCMS is the Huygens probe gas chromatograph and mass spectrometer; hi-res means high spatial resolution; thermal IR means wavelengths in the thermal infrared (for Titan, tens of microns wavelength).

or the International Standard Atmosphere tables). At that point, because of the dependence of the vapor pressure of water on the exponential of $1 / \mathrm{T}$, which $\mathrm{T}$ is temperature, the amount of water vapor that can exist stably is orders of magnitude smaller than at Earth's surface. Where the mixing ratio of water may be typically $0.1-1 \%$ at Earth's surface, it is 10 parts per million - four orders of magnitude lower - at the tropopause [28]. This limits severely the amount of water that is admitted to the stratosphere, where water is transported quickly upward to the mesosphere where (principally at $80-85 \mathrm{~km}$ ), solar ultraviolet radiation breaks water into $\mathrm{O}$ and $\mathrm{OH}$ [29], leading ultimately to loss of hydrogen and hence permanent loss of water. That our ocean has been stable over geologic time, at least after an early period of heavy bombardment by asteroidal and cometary bodies [30], is largely thanks to the extreme cold trap afforded by the low tropopause temperature that severely limits the leak rate of water from troposphere to stratosphere.

Astronomers have ample evidence that the Sun will brighten with time in an essentially monotonic fashion, as hydrogen converts to helium and makes its interior denser. As it does so, the carbon dioxide feedback that partly buffers the increase will eventually cease as the atmospheric carbon dioxide abundance becomes so small as to be inconsequential for radiative balance (and, before that, to sustain photosynthesis). Rising surface temperature will lead 
to increased evaporation of water from the oceans which, in turn, will enhance greenhouse warming. Whether an instability - a runaway - then occurs is unclear because the radiative equilibrium problem can have multiple solutions [31]. However, the question of a runaway is only one of how soon water is lost. The evolution of the solar luminosity makes it almost unavoidable that, within the next few billion years, the tropopause temperature of the Earth will be elevated to the point that loss of the Earth's oceans by evaporation and then stratospheric photolysis of the water vapor will occur over a timescale of order a billion years [32]. From that point on, surface liquid water will be limited to the poles, will have a short photochemical lifetime, and would persist on the surface only if re-supplied by mantle/deep crustal water. At the equator, occasional intense rainstorms might scour out fluvial features, but the principle sedimentary deposits will be sand dunes.

How long such a climate would exist is rendered uncertain by the continued volcanic eruption of carbon dioxide, which in the absence of the oceans and hence significant rainfall might not be converted to carbonates, and could drive the Earth toward a "supergreenhouse" state like that of Venus [34]. On the other hand, most of the carbon dioxide in the crustal and even upper mantle environment might have been converted to carbonates by then, as well as organic molecules much of which might be buried and rendered relatively stable. In the absence of water, plate tectonics will likely cease and prevent carbonate deposits from recycling into the deeper crust to reform carbon dioxide. The Earth might thus retain a dry climate, but one in which small amounts of water were stable at the poles, for some significant length of time.

The closest analog in our solar system to such a future climate for the Earth might be that of Titan's methane cycle illustrated in Fig. 2. Substitute water for methane, the organic dunes with silicate dunes (though one might wonder, in the absence of subduction, whether seafloor carbonate sediments might contribute to the dune fields on such an ocean-less Earth), and the hydrocarbon products of photochemistry with oxygen and ozone, and one has a possible - admittedly speculative - model for our planet's future hydrological cycle. Thus by studying how the various geologic and meteorological processes interact on Titan, we have a peek at the Earth's future "simulated" on a planetary scale with different materials, but operating actively in the present day. At a minimum, with Titan we have the opportunity to see how a "hydrologic" regime very different from that of Earth or Mars might operate on a planet-wide scale.

\section{Next steps in exploring Titan}

The Cassini-Huygens mission is entering its extended mission phase and will make additional observations of Titan for at least the next two years, barring any technical problems. These observations will undoubtedly lead to new discoveries and insights into ones already made. In particular, depending on how long Cassini operates, a significant part of the seasonal cycling of methane from lakes in the summer hemisphere to those in the winter hemisphere might be observed. However, the information already obtained is enough to plan a future mission to Titan with instruments designed to address those important questions raised by the Cassini-Huygens measurements (Fig. 4). This is the way exploration proceeds: a deeper level of understanding afforded by a given mission leads to more complex and detailed questions that could not even be formulated prior to that mission.

Sometime in the future, a probe might splash down into a deep sea of methane and ethane in Titan's northern hemisphere, while a hot air balloon courses across thousands of kilometers of equatorial dunes of solid organics. Overhead, an orbiter maps Titan at 50 meter resolution in the near-infrared ( 5 micron wavelength) part of the spectrum where haze and methane absorption are minimal, profiles the crust with radar to determine the topography of mountains, dunes and river systems, and relays data from the lake lander and balloon. This is the present concept for a follow-on mission to Titan, called the Titan Saturn System Mission (TSSM), which is being considered at the time of writing as a possible joint mission by the US (NASA) and European (ESA) space agencies. The lake lander would carry a sophisticated package to measure the bulk composition of the lake, the abundance of dissolved organics, and the physical properties of 
the liquid. Because many organic compounds are soluble enough in liquid methane and ethane to be detected by a mass spectrometer, the large seas are a convenient reservoir for sampling organics that may have been brought there by the wind from more distant places on Titan. The balloon would map the terrain it overflies, probe for sedimentary layers in the surface organics with long-wavelength radar, determine composition of organic deposits on scales of tens of meters, and being underneath Titan's shielding ionosphere, could search for an elusive internal magnetic field the Cassini Orbiter has failed to find. Together - orbiter, balloon, lake lander - the three components of the mission would allow deep probing of the methane cycle and a search over large areas for complex organics on this fascinating but still enigmatic world.

\section{References}

1. N. Grevesse, M. Asplund, A.J. Sauval, Space Sci. Rev. 130, 105 (2007)

2. G. Tobie, O. Grasset, J.I. Lunine, A. Mocquet, C. Sotin, Icarus 175, 496 (2005)

3. A.D. Fortes, P.M. Grinrod, S.K. Trickett, L. Vocadlo, Icarus 188, 139 (2007)

4. J.I. Lunine, S. Atreya, Nat. Geosci. 1, 159 (2008)

5. R.D. Lorenz, J. Mitton, Titan unveiled: Saturn's mysterious moon explored (Princeton University Press, Princeton, N.J., 2008), p. 296

6. A. Coustenis, F. Taylor, Titan: The Earth-like moon (World Scientific Publishing, Singapore, 2008), p. 400

7. V. Vuitton, R.V. Yelle, M.J. McEwan, Icarus 191, 722 (2007)

8. A. Coustenis, et al., Icarus 189, 35 (2007)

9. R.D. Lorenz, et al., Geophys. Res. Lett. 35, L02206 (2008) doi:10.1029/2007GL032118

10. M. Fulchignoni, et al., Nature 438, 785 (2005)

11. J. Radebaugh, et al., Icarus 194, 690 (2008)

12. E. Stofan, et al., Nature 445, 61 (2007)

13. M. Tomasko, et al., Nature 438, 765 (2005)

14. R.D. Lorenz, et al., Science 319, 1649 (2008)

15. R. Hueso, A. Sànchez-Lavega, Nature 442, 428 (2006)

16. J.I. Lunine, Rev. Geophys. 31, 133 (1993)

17. J.H. Waite, et al., Science 311, 1419 (2006)

18. S. Horst, V. Vuitton, R. Yelle, J. Geophys. Res. (2008) (in press)

19. D.P. O'Brien, R.D. Lorenz, J.I. Lunine, Icarus 173, 243 (2005)

20. D.J. Stevenson, Nature 298, 142 (1982)

21. R.M.C. Lopes, et al., Icarus 186, 395 (2007)

22. C.D. Neish, A. Somogyi, H. Imanaka, J.I. Lunine, M.A. Smith, Astrobiology 8, 273 (2008)

23. D.K. Kondepudi, G.W. Nelson, Nature 314, 438 (1985)

24. S.A. Benner, A. Ricardo, M.A. Carrigan, Curr. Opinion Chem. Biol. 8, 672 (2004)

25. R.H. Brown, et al., Nature 454, 607 (2008)

26. P. Paillou, et al., Geophys. Res. Lett. 35, CiteID L05202 (2008)

27. Committee on the Origin and Evolution of Life, The limits of organic life (National Academy Press, Washington, DC, 2007), p. 100

28. K.H. Rosenlof, Science 302, 1691 (2003)

29. I.M. Vardavas, J.H. Carver, F.W. Taylor, Ann. Geophys. 16, 189 (1998)

30. N.H. Sleep, K.J. Zahnle, J.F. Kasting, H.J. Morowitz, Nature 342, 139 (1989)

31. T. Pujol, G.R. North, J. Atmos. Sci. 59, 2801 (2002)

32. K. Caldeira, J.J. Kasting, Nature 360, 721 (1992)

33. R.T. Pierrehumbert, J. Atmos. Sci. 52, 1784 (1995)

34. J. Kasting, Icarus 74, 472 (1988)

35. Solar System Exploration Survey, Frontiers in the solar system: An integrated exploration strategy (National Academy Press, Washington DC, 2003), p. 248 